\section{Flammenatomabsorptionsspektro- metrie/-spektroskopie}

J. Knecht

FB Chemie; Division.:Analytical Chemistry, Universität

Marburg, Marburg, Deutschland

Synonym(e) Flammenatomabsorptionsspektroskopie;

Flammen-AAS

Englischer Begriff flame atomic absorption spectrometry

Definition Bei der Flammenatomabsorptionsspektrometrie werden die zu bestimmenden Atome mit einer heißen Flamme erzeugt.

Beschreibung Bei der Flammen-AAS wird eine Lösung (z. B. Patientenprobe) des zu bestimmenden Elements als Aerosol in die Flamme (entweder Luft-Acetylen $\left[\mathrm{C}_{2} \mathrm{H}_{2}\right]$ $\left[2400{ }^{\circ} \mathrm{C}\right]$ oder Lachgas- $\left[\mathrm{N}_{2} \mathrm{O}-\right]$ Acetylen $\left.\left[2800{ }^{\circ} \mathrm{C}\right]\right)$ gesaugt und dort versprüht. Hierbei laufen folgende Schritte ab:

1. Desolvatation des Aerosols (das Wasser der Lösung wird verdampft, zurück bleiben feste Teilchen)

2. Verdampfung der festen Teilchen zu Molekülen

3. Dissoziation der Moleküle in Atome

4. Teilweise Ionisation der Atome (unerwünscht, da nur die Konzentration der Atome in der Flamme gemessen werden kann)
5. Reaktion der Atome mit anderen Teilchen, die in der Flamme sind (unerwünscht, da die Konzentration an freien Atomen in der Flamme verringert wird).

Das von der elementspezifischen $>$ Hohlkathodenlampe oder elektrodenlosen Entladungslampe ausgehende Licht wird nicht nur von den Atomen des zu bestimmenden Elements sondern auch unspezifisch durch den kontinuierlichen Untergrund geschwächt. Um die das Messsignal störende unspezifische Untergrundabsorption zu beseitigen, wird diese entweder mit einem Kontinuumstrahler (meist mit einer $>$ Deuteriumlampe) oder mit der sog. Zeeman-Untergrundkompensation (s. - Untergrundkompensation) korrigiert.

In neuerer Zeit wird manchmal die Fließinjektionsanalyse mit der Flammen-AAS gekoppelt, um diese gerade im Routinebetrieb noch vielseitiger einzusetzen. Damit ermöglicht man

- Online-Aufkonzentrierung des zu bestimmenden Elements, um die Nachweisgrenzen zu erniedrigen,

- Verdünnungen, um den linearen Bereich zu erweitern, und

- die teilweise Entfernung der Matrix, um deren Störungen zu verringern. Details unter Atomabsorptionsspektrometrie.

\section{Literatur}

Van Loon JA (2012) Analytical atomic absorption spectroscopy: selected methods, Elsevier, Amsterdam

Welz B, Sperling M (1997) Atomabsorptionsspektrometrie, 4. Aufl. Wiley-VCH, Weinheim 